\title{
Advances in quantum waveguide dynamics, with applications to physical and biological nanostructures
}

\author{
IAIN JOHN CLARK
}

It is known $[\mathbf{1}, \mathbf{2}]$ that as semiconductor microstructures get progressively smaller and more free of impurities, it becomes increasingly necessary to use quantum mechanics to describe accurately these systems, which may have features sized between a few and several hundred nanometers. Recent advances in fabrication technology [11] make it feasible to build such nanostructures or mesoscopic systems, as they are known.

The term "quantum waveguide" [6] is used to denote a nanostructure which acts as a conducting wire, along which a wavefunction is guided to move by the boundary conditions. Unlike classical wires, curved quantum waveguides have curvature-induced bound states; see [6] for a recent review. This has implications for nanoelectronic circuit design, since the curvature-induced bound states will appear as resonances. However, the effect of torsion (where the waveguide twists out of a plane) and twisting (where the waveguide changes its orientation along its path) upon bound states has attracted little investigation, and is the motivation for the analysis in this work.

This thesis develops a unified mathematical approach by which the energy spectrum of a quantum waveguide with curvature and torsion can be analysed, for any crosssectional geometry that remains uniform along the waveguide.

Following the introduction and literature review of Chapter 1 , a non-relativistic Hamiltonian $H$ for a particle on a quantum waveguide is set up in Chapter 2. A system of curvilinear coordinates is used to express Schrödinger's equation using the LaplaceBeltrami operator $\nabla^{2}$. The metric tensor $G$ is expressed as the product of a curvaturedependent matrix $F$ and the metric tensor $\check{G}$ for a "straightened-out" waveguide. The Hamiltonian $H$ is then shown to be unitarily equivalent to the Hamiltonian $H_{U}$ for a straightened-out waveguide plus interaction terms, $H_{U}=H_{0}+K_{U}+V_{U}$, where $H_{0}$ is the Hamiltonian for a "straightened-out" waveguide, $K_{U}$ is a kinetic energy perturbation term and $V_{U}$ is an effective potential.

In Chapter 3, the spectral theory needed to investigate the curvature-induced bound states is developed. The spectrum of a Hamiltonian is partitioned into the

Received 19th June, 1997

Thesis submitted to the University of Queensland, December 1996. Degree approved July, 1997. Supervisor: Professor A.J. Bracken.

Copyright Clearance Centre, Inc. Serial-fee code: 0004-9729/97 \$A2.00+0.00. 
discrete spectrum (corresponding to bound states) and the essential spectrum (corresponding to free states). Since $H$ and $H_{U}$ are unitarily equivalent, they have the same spectra. Under quite strong decay conditions on the curvature of the waveguide, geometric spectral analysis is used to show that the essential spectra of $H$ and $H_{0}$ coincide. A necessary and sufficient condition is then given for the existence of bound states.

Chapter 4 applies the methods of Chapters 2 and 3 to tubular quantum waveguides, which have circular cross-section, and it is shown that the results obtained are in agreement with those of $[\mathbf{7}, \mathbf{8}]$. Further, it is shown that the imposition of torsion weakens the argument for the existence of curvature-induced bound states [5] and raises the energy values for these bound states.

In Chapter 5, the methods of this thesis are applied to quantum strip waveguides, which do not have rotational symmetry in the transverse directions. Consistency with previous work on planar quantum strips [9] is demonstrated, and it is shown [4] that the imposition of twisting and torsion reduces the attractive strength of the effective potential and introduces a repulsive term.

Chapter 6 presents a preliminary investigation into the effects of curvature on the dynamics of wave propagation along microtubules, which are a form of biological nanostructure that is suspected to play a key role in consciousness [10]. It is argued that geometric effects need to be considered in any model of microtubular dynamics [3], since the variations in geometry can be expected to lead to dispersion in propagating waves. An elementary model is developed which attempts to describe quantum coherent phenomena along microtubules.

Finally, in Chapter 7, an overview of the thesis is given together with some ideas for continuing research.

\section{REFERENCES}

[1] J.R. Barker and D.K. Ferry, 'On the physics and modeling of small semiconductor devices', Solid-St. Electron. 23 (1980), 519-530.

[2] F.A. Buot, 'Mesoscopic physics and nanoelectronics: nanoscience and nanotechnology', Phys. Rep. 234 (1993), 73-174.

[3] I.J. Clark, 'The geometric curvature of microtubules may play a part in information processing', Bioelectrochemistry and Bioenergetics 41 (1996), 59-61.

[4] I.J. Clark and A.J. Bracken, 'Effective potentials of quantum strip waveguides', J. Phys. A 29 (1996), 339-348.

[5] I.J. Clark and A.J. Bracken, 'Bound states in tubular quantum waveguides with torsion', J. Phys. A 29 (1996), 4527-4535.

[6] P. Duclos and P. Exner, 'Curvature induced bound states in quantum waveguides in two and three dimensions', Rev. Math. Phys. 7 (1995), 73-102.

[7] P. Exner, 'Quantum waveguides : energy bounds and critical thickness', in Rigorous results 
in Quantum dynamics, (J. Dittrich and P. Exner, Editors) (World Scientific, Singapore, 1991), pp. 125-142.

[8] P. Exner, 'Bound states in quantum waveguides of a slowly decaying curvature', J. Math. Phys. 34 (1993), 23-28.

[9] P. Exner and P. Šeba, 'Bound states in curved quantum waveguides', J. Math. Phys. 30 (1989), 2574-2580.

[10] S.R. Hameroff and R. Penrose, 'Orchestrated reduction of quantum coherence in brain microtubules - a model for consciousness', Math. Comput. Simulation 40 (1996), 453-480.

[11] M.J. Kelly, Low dimensional semiconductors: materials, physics, technology, devices (Clarendon Press, Oxford, 1995).

Department of Mathematics

The University of Queensland

Queensland 4072 Australia

e-mail: ijc@maths.uq.edu.au 